\title{
The nurse's role in feeding patients with renal disease
}

\author{
Elaine P. Murray \\ Haemodialysis Unit, Royal Free Hospital, London NW3 2QG, UK
}

\begin{abstract}
Malnutrition in chronic haemodialysis patients has been widely documented (Leung \& Dwyer, 1998; Milano et al. 1998). Malnutrition in this patient group is often associated with increased morbidity and mortality and decreased quality of life. There are many contributing factors, including inadequate dialysis, nutrient losses during dialysis, dietary restrictions, medications and socioeconomic factors. It is the role of the renal nurse to identify patients at risk from malnutrition and make appropriate referral to the dietician.

Intradialytic parenteral nutrition (IDPN) is one proposed treatment for malnutrition. However, before patients
\end{abstract}

commence IDPN it is essential that all other nutrition strategies should be considered. For IDPN to be successful, patients must be well dialysed and have a minimum of $4 \mathrm{~h}$ dialysis three times per week. These patients will require additional monitoring by nurses before commencing dialysis, during treatment and after completion of treatment. To achieve a successful outcome, a multidisciplinary approach is required, with all disciplines sharing information to improve patient care.

A prospective study of patient outcome following use of IDPN is proposed for the future in our renal unit.

Intradialytic parenteral nutrition: Renal disease: Malnutrition

\section{References}

Leung J \& Dwyer J (1998) Renal DETERMINE nutrition screening tools for the identification and treatment of malnutrition. Journal of Renal Nutrition 8, 95-103.
Milano MC, Cusumano AM, Navarro ET \& Turin M (1998) Energy supplementation in chronic hemodialysis patients with moderate and severe malnutrition. Journal of Renal Nutrition $\mathbf{8}$, 212-217.

(C) Nutrition Society 2000

Abbreviation: IDPN, intradialytic parenteral nutrition.

Corresponding author: Ms Elaine Murray, fax +44 (0)20 7830 2125, email ElaineMurray @rfh.nthames.nhs.uk 